\title{
Highly individual SWCNTs for high performance thin film electronics
}

Antti Kaskela ${ }^{1, *}$, Patrik Laiho ${ }^{1}$, Norihiro Fukaya ${ }^{2}$, Kimmo Mustonen $^{1}$, Toma Susi ${ }^{3}$, Hua Jiang ${ }^{1}$, Nikolay Houbenov ${ }^{1}$, Yutaka Ohno ${ }^{2}$ and Esko I. Kauppinen ${ }^{1, *}$.

${ }^{1}$ Department of Applied Physics, Aalto University School of Science, P.O. Box 15100, FI-00076 Aalto, Finland

${ }^{2}$ Department of Quantum Engineering, School of Engineering, Nagoya University, Furo-cho, Chikusa-ku, Nagoya 464-8603, Japan

${ }^{3}$ University of Vienna, Faculty of Physics, Boltzmanngasse 5, 1090 Vienna, Austria

*Corresponding author:

Dr. Antti Kaskela, antti.kaskela@gmail.com

Prof. Dr. Esko I. Kauppinen, esko.kauppinen@aalto.fi 


\begin{abstract}
We report a continuous floating catalyst chemical vapor deposition synthesis of highly individual single-walled carbon nanotubes (SWCNT) for high performance transparent conducting films (TCF). Active feedback dilution of ferrocene-based catalyst vapor leads to an almost complete elimination of SWCNT bundling and a substantial increase in SWCNT lengths via the suppression of bundling-induced growth termination. The fabricated uniform TCFs exhibit sheet resistances of $89 \Omega /$ sq. at $90 \%$ transmittance. This was further improved by micro-patterning, resulting in a sheet resistance of $69 \Omega / \mathrm{sq}$. at $97 \%$ transmittance - the highest reported for any carbon nanotube TCF and highly competitive with commercial indium-tin-oxide-TCFs. Furthermore, we demonstrate that thin film transistors fabricated from these highly individual SWCNTs reach charge carrier mobilities up to $100 \mathrm{~cm}^{2} \mathrm{~V}^{-1} \mathrm{~s}^{-1}$ and ON/OFF-ratios up to $10^{6}$.
\end{abstract}




\section{Introduction}

Single-walled carbon nanotubes (SWCNT) have exceptional application potential in thin film electronics due to their unique electrical and optical properties, which can be exploited in variety of applications, including transparent conductive films (TCFs) ${ }^{[1-7]}$ and thin film transistors $(\mathrm{TFTs})^{[8-13]}$. SWCNT networks can be fabricated using a variety of techniques, such as spray coating and press transfer, and they exhibit high flexibility, high maximum transmittance and low sheet resistance ${ }^{[6,7,14]}$. SWCNT networks have been also used to fabricate TFTs via transfer processing of dry fabricated SWCNT networks ${ }^{[12-13]}$ or by inkjet and gravure printing ${ }^{[15-16]}$, resulting in high charge carrier mobilities and ON/OFF current ratios. Combined, these enable the potentially high throughput fabrication of SWCNT based flexible integrated circuits. Almost all SWCNT synthesis processes, such as arc discharge, laser ablation and HiPCO, produce a mixture of SWCNTs, bundles, by-products and impurities, necessitating extensive purification and dispersion treatments before their use in electronic applications ${ }^{[17]}$. These are detrimental for performance, as high power ultrasonication induces defects and shortens SWCNTs, while surfactants used for aqueous dispersion can be difficult to remove, leading to high contact resistances and limited device performance $^{[2,18-20]}$. Dispersion of SWCNT bundles is also essential prior to liquid phase helicity separation techniques, such as density gradient ultracentrifugation ${ }^{[21]}$ or gel chromatography ${ }^{[22]}$, which can be used to separate semiconductive and metallic SWCNTs. Elimination of these processing steps is necessary for the industrial production of SWCNT based components. For ultimate efficiency, synthesis and deposition processes must be developed to enable the direct fabrication of individualized SWCNTs with narrow chirality distributions.

Floating catalyst chemical vapor deposition (FC-CVD) is a promising SWCNT synthesis technique with reduced dependency on liquid processing ${ }^{[23]}$. It enables the direct, single-step fabrication of clean and ready-to-use SWCNT networks. The process has been used for the fabrication of high performance SWCNT TCFs ${ }^{[6,7]}$ and TFTs ${ }^{[12-13]}$. Previous studies have shown that the SWCNT and bundle morphologies have a major impact on the performance of SWCNT networks ${ }^{[6,12,24]}$. First, increasing bundle length correlates with decreasing sheet resistance at a constant optical transparency, as longer bundles reduce the amount of high resistance inter-bundle contacts in the network ${ }^{[6]}$. Second, the importance of long, extended y-type contacts and small diameter bundles has been demonstrated in the context of low- 
density SWCNT networks for $\mathrm{TFTs}^{[12]}$ and by spatially resolved conductive-AFM characterization of inter-tube contacts ${ }^{[24]}$. Third, individual SWCNTs are also indispensable for the efficient electrostatic gate-channel coupling in SWCNT thin film transistors ${ }^{[12]}$, with reduced SWCNT bundling also helping to decrease the probability of metallic short circuited channels in SWCNT TFTs ${ }^{[25]}$. Finally, gas phase bundling is expected to lead to SWCNT growth termination, and thus for optimal SWCNT TCF performance requiring long SWCNTs, bundling should be reduced or completely eliminated.

\section{Experimental}

Our SWCNT synthesis process is based on the low vapor pressure organometallic compound, ferrocene, which is sublimated in a flow of carbon monoxide (CO). The ferrocene-containing gas is injected through a water-cooled injector probe, maintained at a constant temperature of $24{ }^{\circ} \mathrm{C}$, into a high temperature synthesis reactor whose maximum temperature is $880{ }^{\circ} \mathrm{C}$ based on previous process optimization ${ }^{[6]}$. The ferrocene is thermally decomposed to iron vapor in the steep temperature gradient immediately after exiting the water-cooled injector. The iron vapor subsequently nucleates into iron nanoparticles, which catalyze the SWCNT growth by the Boudouard reaction in the laminar flow inside the synthesis reactor. The reactor chamber consists of an inert-walled quartz tube of $560 \mathrm{~mm}$ in length and $22 \mathrm{~mm}$ in diameter. The grown SWCNTs can be collected at the reactor outlet by membrane filtration as randomly oriented SWCNT networks, which have been previously shown ${ }^{[6]}$ to primarily consist of small diameter SWCNT bundles around $10 \mathrm{~nm}$ in diameter.

The probability for aerosol particles to collide, leading to bundling, has a quadratic dependence on the particle concentration ${ }^{[26]}$,

$$
\frac{d N}{d t}=-K_{0} N^{2}
$$

where $\mathrm{N}$ is the aerosol number concentration and $\mathrm{K}_{0}$ is the size and temperature dependent coagulation coefficient. This suggests that collisions between SWCNTs can be suppressed by reducing the number concentration of the SWCNT aerosol. The catalyst particle concentration and subsequent SWCNT concentration depend on the amount of ferrocene introduced into the reactor. Here, the ferrocene cartridge was placed in a water-cooled heat 
bath maintained at $24^{\circ} \mathrm{C}$ to maintain a stable catalyst precursor vapor pressure. To further improve concentration control, we developed an on-line monitoring and feedback system, schematically depicted in Figure 1. The system is based on microprocessor-controlled mass flow controllers (GFCS series, Aalborg Instruments, USA) for the controllable mixing of ferrocene-containing $\mathrm{CO}$ with pure $\mathrm{CO}$ for catalyst concentration adjustment, together with fast aerosol monitoring at the reactor outlet using a differential mobility analyzer and a condensation particle counter (5414 CPC with medium length Vienna type DMA, Grimm Aerosol Technik GmbH). The system improves the reproducibility and stability of the SWCNT synthesis process, enabling rapid feedback of the synthesis process. For concentration-controlled FC-CVD synthesis, a minor fraction of the CO flow was passed through the ferrocene saturator (volumetric flow of 50-100 ccm) and mixed with pure CO downstream of the saturator cartridge (200-250 ccm). To maintain constant particle concentration, the mixing ratio of ferrocene-saturated and pure CO flows was varied while maintaining the total main flow at $300 \mathrm{ccm}$. Figure $1 \mathrm{~b}$ shows the acquired SWCNT number size distributions at different ferrocene concentrations, measured by the DMA system probing the floating structure concentration vs. electrical mobility diameter $D_{p}$. The feedback system significantly improved the temporal stability of the FC-CVD process, leading to less than $10 \%$ standard deviation in concentration over 40 hours (Figure 1c). Interestingly, high resolution TEM (HR-TEM) imaging in a JEOL JEM-2200FS operated at 80kV (Figure $1 \mathrm{~d}$-f) suggests that the increasing ferrocene concentration leads to increasing bundling, clearly observable in samples directly deposited on TEM grids at the reactor outlet. 

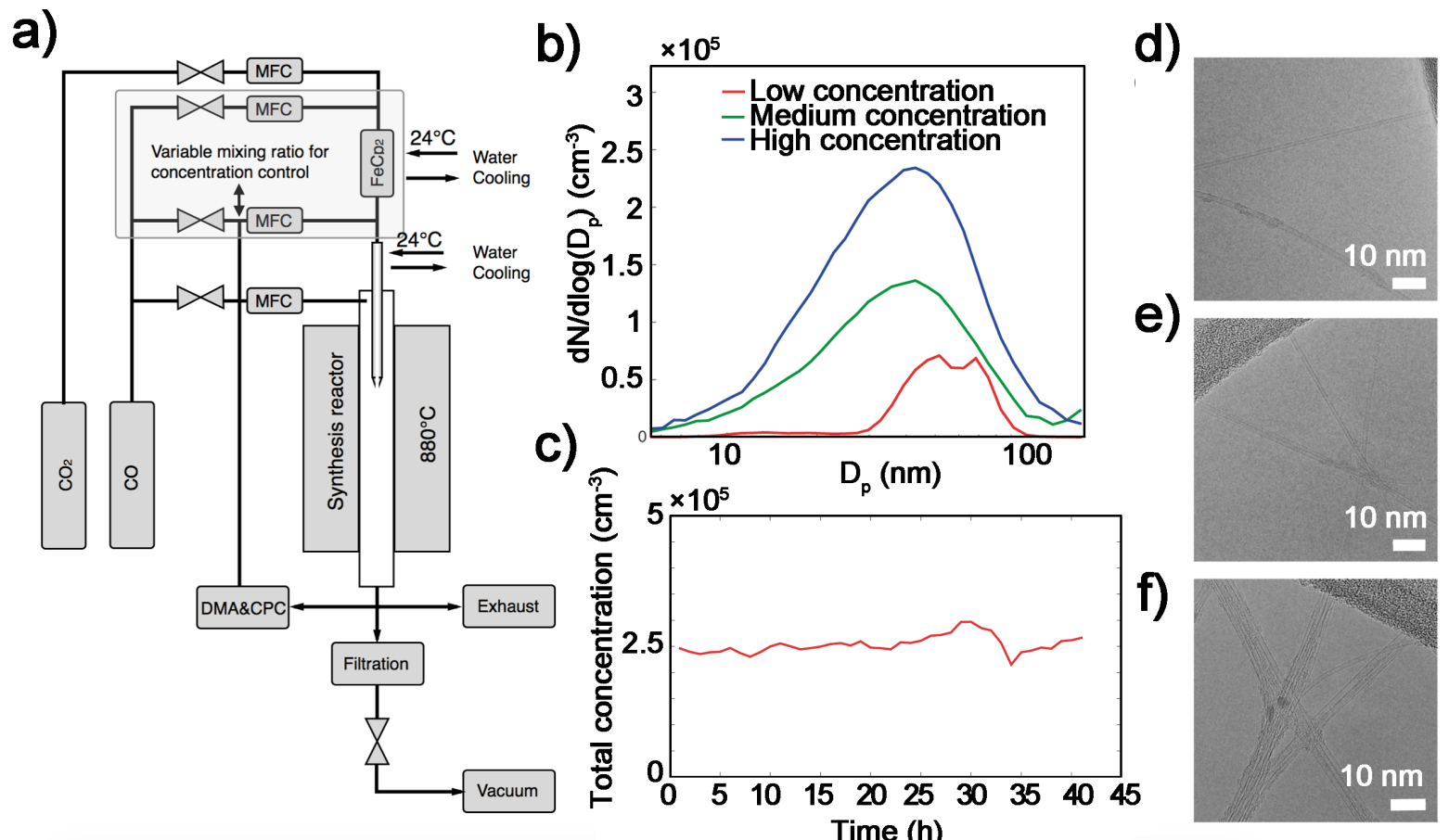

Figure 1. a) The FC-CVD SWCNT synthesis reactor includes temperature control of the ferrocene cartridge to maintain a stable precursor vapor pressure. Feedback-controlled mixing further improves control of the catalyst precursor and subsequent catalyst particle and SWCNT concentrations in the reactor. b) Number size distributions from high to low ferrocene concentrations, showing a substantial decrease in total particle concentration from $2.0 \times 10^{6} \mathrm{~cm}^{-3}$ via $1.2 \times 10^{6} \mathrm{~cm}^{-3}$ to $0.25 \times 10^{6} \mathrm{~cm}^{-3}$. c) Particle concentration feedback improves reactor stability, reaching $<10 \%$ standard deviation in total particle concentration at the reactor outlet over $40 \mathrm{~h}$ of continuous synthesis. d-f) HR-TEM imaging for low, medium and high ferrocene concentrations, respectively, suggests that increasing ferrocene concentration leads to increasing bundling in samples directly deposited on TEM grids.

For this work, the FC-CVD process was operated at three specific conditions: 1) Low concentration, where approximately $50 \mathrm{ccm}$ of $\mathrm{CO}$ was passed through the ferrocene cartridge, leading to a total particle concentration of $0.25 \times 10^{6} \# / \mathrm{cm}^{3}$; 2) Medium concentration, $75 \mathrm{ccm}$ flow through the cartridge, particle concentration $1.2 \times 10^{6} \mathrm{\#} / \mathrm{cm}^{3}$; and 3) high concentration, $100 \mathrm{ccm}$ flow through the cartridge, particle concentration $2 \times 10^{6}$ $\# / \mathrm{cm}^{3}$. The total particle concentrations were deduced by integrating the area under normalized number size distributions shown in Figure 1b. Randomly oriented SWCNT networks from each condition were deposited on membrane filters and subsequently transferred by room-temperature press transfer $^{[6]}$ onto high transparency quartz slides (HSQ300, Heraeus GmbH) for optical spectroscopy and electrical characterization, or to 
flexible polyethylene naphthalate substrates (PEN 65FA, $100 \mu \mathrm{m}$, Teijin-DuPont, Japan) for flexible TCF fabrication. The absorbance measurements were carried out using PerkinElmer Lambda 950 spectrometer (PerkinElmer, Inc., USA) and sheet resistances were acquired using a Jandel Ltd. 4-point probe at 60-gram needle loading and $250 \mu \mathrm{m}$ tip radius for the uniform SWCNT TCFs.

For the line grid patterned SWCNT TCFs the sheet resistance was estimated by depositing silver paint (Leitsilber 200N) contacts at the edges of a square are covered by the grid pattern of $10 \mathrm{~mm}$ x $10 \mathrm{~mm}$ and by performing 2-terminal resistance measurement by using Agilent 34410A multimeter at moderate measurement current of $10 \mathrm{~mA}$, to avoid excessive Ohmic heating. The current was supplied in direction parallel to the horizontal lines in the Figure 4c, and the measured grid area was limited by mechanical cutting along the measurement area edges. Electrical contacts to the sample were formed by using Suss Microtech PA150 probe station equipped with tungsten carbide needles on precisely controllable micromanipulator for stable and low resistance mechanical contact to the silver paint contact areas. The optical transparency was measured by limiting the beam size to $10 \mathrm{~mm}$ x $5 \mathrm{~mm}$ area which was completely covered by the grid pattern, thus effectively measuring the average transparency over the grid pattern.

Our results show that lower ferrocene concentrations lead to substantially improved TCF performance. Pristine, as-collected SWCNT TCFs from the high and medium concentration conditions exhibited sheet resistances of 5 and $1.3 \mathrm{k} \Omega / \mathrm{sq}$. at $90 \%$ transmittance, whereas the low concentration leads to SWCNT TCFs of $950 \Omega /$ sq. (Figure 2a). Chemical doping is a well-established method to further improve the sheet resistance of SWCNT networks by charge-transfer induced Fermi level shifting and subsequent increase in charge carrier density ${ }^{[4],[27]}$. To further improve TCF performance and to enable direct comparison with our previous work, we used a rapid post-deposition treatment, consisting of densification and wetting SWCNT network by concentrated nitric acid (65 \% $\mathrm{HNO}_{3}$, J.T. Baker, Netherlands) for $60 \mathrm{~s}^{[6]}$. The acid treatment reduced the sheet resistances of the high, medium and low concentration films to 440, 120 and $89 \Omega /$ sq. at $90 \%$ transmittance, respectively. Interestingly, SWCNT networks collected from the low concentration conditions exhibited highly resolved $E_{11}$ and $E_{22}$ peaks in optical absorption spectroscopy. These peaks are related 
to optical transitions between the first and second van Hove singularities of semi-conductive SWCNTs, indicative either of a narrow SWCNT chirality range or reduced bundling.

a)

b)
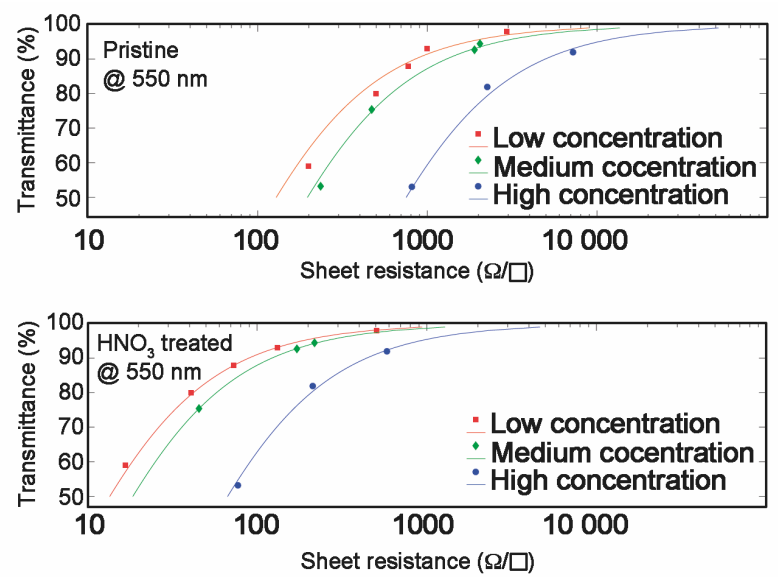

d)
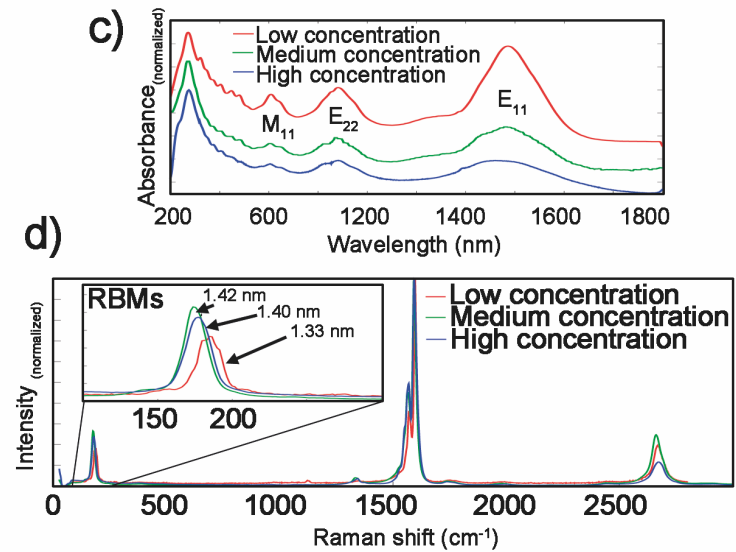

Figure 2. Sheet resistance vs. transmittance of SWCNT TCFs a) before and b) after chemical doping with $\mathrm{HNO}_{3}$. Number concentration reduction yields a systematic improvement in TCF performance with sheet resistances of $5,1.3$ and $0.95 \mathrm{k} \Omega / \mathrm{sq}$. at $90 \% \mathrm{~T}$, for high, medium and low concentration. The trend is maintained after the rapid $\mathrm{HNO}_{3}$ chemical doping, yielding sheet resistances of 440, 120 and $89 \Omega /$ sq. at $90 \%$, respectively. c) Optical absorption spectroscopy suggests mean SWCNT diameters of $1.3 \mathrm{~nm}$, with highly resolved $\mathrm{E}_{11}$ and $\mathrm{E}_{22}$ transitions for the lower concentration samples, indicating either a narrow SWCNT chirality range or reduced bundling. d) Raman spectroscopy, presented here for a $514 \mathrm{~nm}$ excitation laser, shows high G/D ratios up to 40, confirming the high quality of the FC-CVD SWCNTs.

Microscopic analysis reveals major differences in SWCNT bundling and network morphologies between samples produced at low and high concentration conditions. First, AFM micrographs (Veeco Dimension 5000 with Bruker TESP-SS cantilever, operated at tapping mode with low drive amplitude to minimize nanotube deformation ${ }^{[30]}$ ) of low density SWCNT networks, collected directly onto exfoliated mica substrates using a thermophoretic precipitator, show that the networks dominantly consist of individual SWCNTs. $77 \%$ of the line profiles, measured every $200 \mathrm{~nm}$ across SWCNTs and bundles, have heights smaller than $1.8 \mathrm{~nm}$, with the mean height of the network estimated at $1.20 \mathrm{~nm}$ as depicted in Figure 3. Considering their mean diameter is $1.3 \mathrm{~nm}$, this indicates that most of the SWCNTs are individual. The medium and high concentration samples exhibit contrasting characteristics caused by increased gas phase bundling: in the medium concentration sample, the fraction of profiles with heights below $1.8 \mathrm{~nm}$ is $57 \%$ with an increased mean height of $1.61 \mathrm{~nm}$, and correspondingly $51 \%$ and $2.36 \mathrm{~nm}$ for the high concentration sample. The tail of the height 
distribution is also lengthened as the concentration increases: in the low concentration sample, the largest observed profile height was $3.5 \mathrm{~nm}$, while the corresponding values for the medium and high concentration are $7.17 \mathrm{~nm}$ and $15.49 \mathrm{~nm}$.

These observations strongly support the hypothesis that gas phase bundling can be suppressed by controllably lowering the catalyst feedstock concentration introduced into the synthesis reactor. Furthermore, the length statistics estimated from scanning electron micrographs (SEM, Zeiss VP, operated at $1.5 \mathrm{kV}$ with an in-lens electron detector) suggest that the lower ferrocene concentration not only leads to reduced bundling, but also yields longer conductive structures with a mean length of $4.8 \mu \mathrm{m}$, while the medium concentration results in a mean length of $4.2 \mu \mathrm{m}$ (Figure $3 \mathrm{~g}-\mathrm{i}$ ). The shorter mean length of $2.9 \mu \mathrm{m}$ at high concentration is likely due to bundling-induced growth termination. We also believe the greater electrical mobility diameter observed at lower number concentration arises from substantially higher average structure lengths (c.f. Figure $1 \mathrm{~b}$ and 3 g-i). It is worth noting that the morphological changes of the SWCNT networks caused by the changes in the catalyst feedstock concentration suggest two separate avenues for further TCF performance improvement. The increased length of the conductive elements of the network, i.e. bundles and individual SWCNTs, leads to a reduced number of highly resistive inter-bundle contacts between SWCNTs per unit length, reducing resistance in direct proportion to the SWCNT length. Second, as the bundle diameter decreases, the amount of parallel conductive pathways leads to an overall sheet resistance inversely dependent on bundle diameter, assuming that the diameter does not have a strong effect on inter-bundle contact resistances ${ }^{[24]}$. 
a)

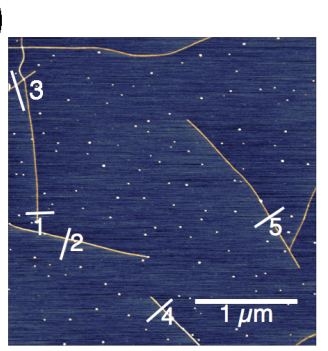

b)

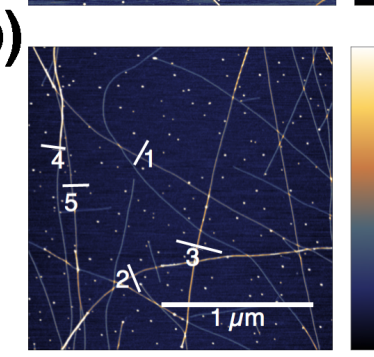

c)

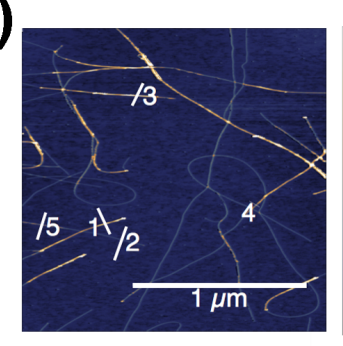

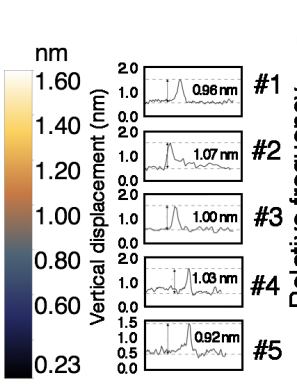
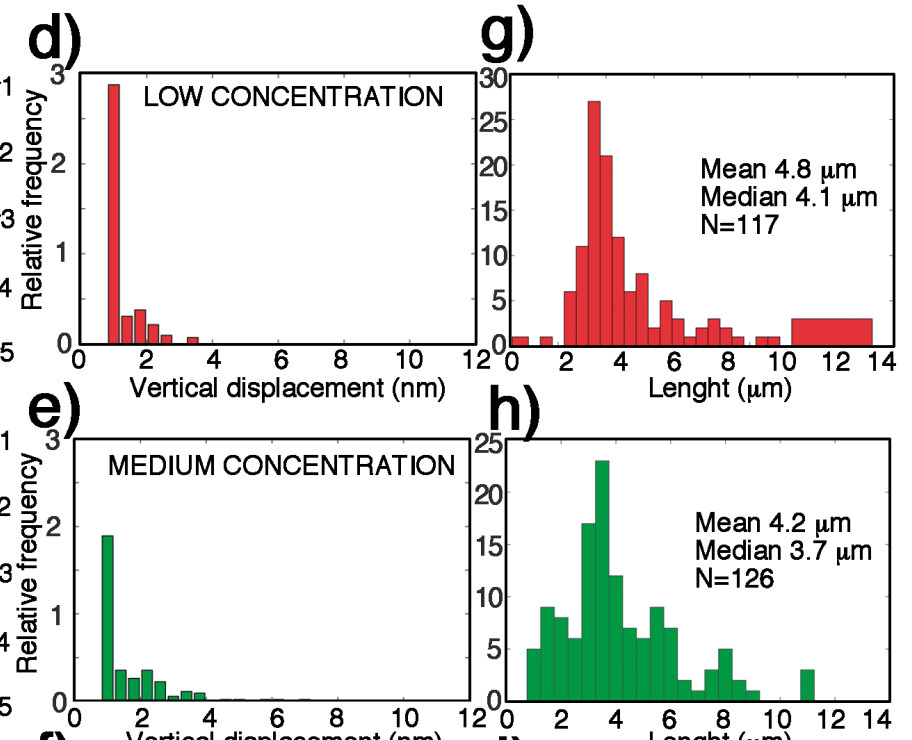

h)

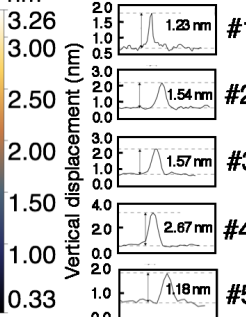

$\mathrm{nm}$
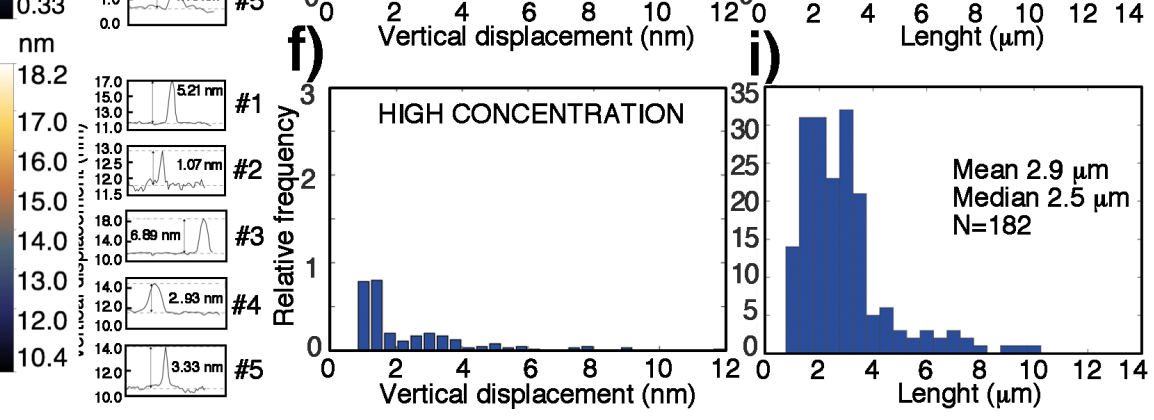

Figure 3. a-c) Low, medium and high concentration AFM micrographs, depicting the typical morphologies and height profiles for SWCNTs or bundles. d-f) Statistical analysis of individual SWCNTs or bundle height profiles estimated from the AFM micrographs show that bundling can be suppressed by reducing the ferrocene concentration, leading to higher fraction of individual tubes. g-i) Individual SWCNTs or bundle length statistics estimated by SEM imaging show that the reduced concentration leads to a substantial increase of median length, ranging from 2.9 to 4.2 to $4.8 \mu \mathrm{m}$ for the high, medium and low concentrations, respectively.

To further improve the performance SWCNT TCFs, we performed patterning by photolithographically fabricated grid pattern on the membrane filters before deposition ${ }^{[28]}$. By selectively depositing SWCNT only on desired locations defined by micro-patterned hydrophobic and solvent-resistant polyvinylidene difluoride (PVDF) filters (Millipore HVHP04700, pore size of $0.45 \mu \mathrm{m}$ ), the overall transparency of the TCF can be increased by increasing the non-SWCNT coated area, while depositing a dense SWCNT network to form a microgrid. We used a parallel line pattern with approximately $8 \mu \mathrm{m}$ wide SWCNT traces at 50, 100, 200 and $400 \mu \mathrm{m}$ spacings, connected with perpendicular crossbars at $2 \mathrm{~mm}$ intervals. 
Flexible micro-grid-patterned SWCNT TCFs with four different grid patterns are shown in Figure 4a. Patterning substantially improves TCF performance as shown in Figure 4b, which displays a comparison with the uniform films mentioned earlier, leading to a highest performance of $69 \Omega /$ sq. at $97 \%$ after $\mathrm{HNO}_{3}$ doping - the highest performance reported for any flexible SWCNT TCF, as shown in Figure 4b.

Networks of highly individualized SWCNTs can be also used as thin film transistor channels. For SWCNT TFT fabrication, we deposited low density $\left(0.6 \# / \mu m^{2}\right)$ SWCNT networks on nitrocellulose membrane filters, subsequently transferred onto $\mathrm{p}^{++}$silicon wafers with a 100 $\mathrm{nm}$ thermal oxide layer and prefabricated source and drain gold electrodes by using the filter dissolution method ${ }^{[12]}$. The transfer process was followed by photolithographic patterning via an etch mask, followed by oxygen plasma reactive ion etching to define the channel area. Output and transfer characteristics were evaluated by a semiconductor parameter analyzer (Agilent 4156B) for 40 SWCNT TFTs with channel dimensions of $100 \times 100 \mu m$. Typical output and transfer characteristics of as-fabricated TFTs are depicted in Figure 5, with a clear transition to saturation regime and high transconductance. Performance parameters, i.e. ON/OFF current ratio and charge carrier mobility, were extracted from the transfer curves using the formula

$$
\mu=\frac{L_{C h}}{W_{C h}} \frac{1}{C} \frac{1}{V_{D S}} \frac{\mathrm{d} I_{D}}{\mathrm{~d} V_{G S}}
$$

for mobility estimation, while using $\mathrm{V}_{\mathrm{DS}}$ of $-1.0 \mathrm{~V}$. It is known that for low density SWCNT network TFTs, the channel capacitance deviates substantially from the parallel plate estimate, and that the network morphology should taken into account by estimating the gate capacitance using a rigorous model ${ }^{[29]}$. As shown in Figure 5, individualized SWCNT can be used to fabricate arrays of SWCNT TFTs with ON/OFF ratios up to $10^{6}$ and mobilities up to $100 \mathrm{~cm}^{2} /$ Vs. The results indicate that the performance realized with the highest individual SWCNT fraction approaches those of previously obtained by Sun et.al. ${ }^{[12]}$, for small diameter and long SWCNT forming the transistors channels, thus providing further evidence of the high individual SWCNT fraction obtained. However, our improved control over bundle diameter via concentration control opens up new experimental directions for evaluation of the effects of bundling for SWCNT TFT performance in a dedicated, future work. 


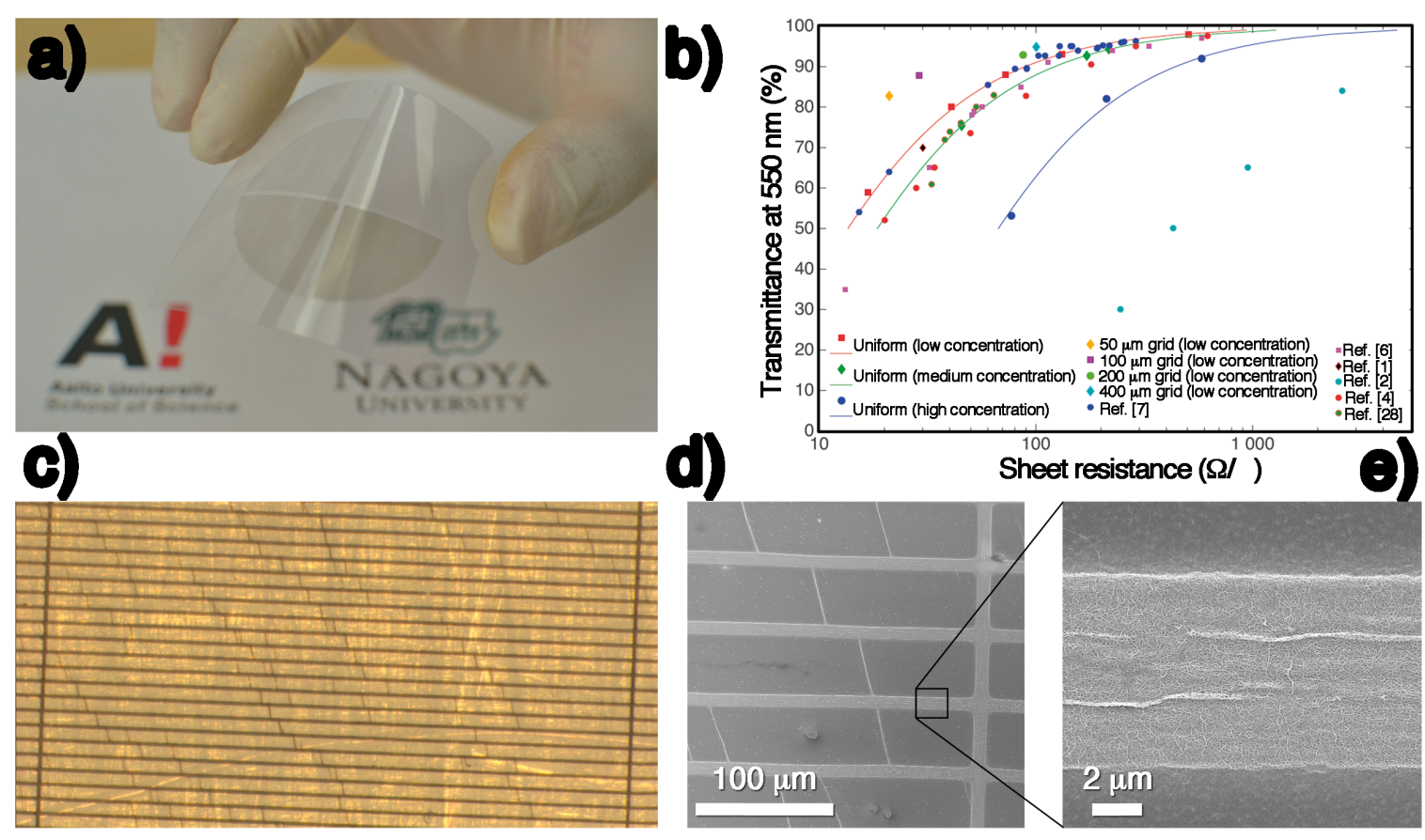

Figure 4. a) Photograph of micro-grid patterned SWCNTs on a PEN substrate, with four different grid designs leading to distinctive transparencies. The micro-patterned TCFs look completely uniform at typical viewing distances of 30-60 cm. b) SWCNT TCF performance comparison of micro-patterned and uniform SWCNT TCFs along with reference data, showing that the highly individual SWCNT films are competitive with other SWCNT-based TCFs. The micro-patterned TCFs fabricated at low concentration have to our knowledge the best SWCNT-TCF performance ever reported. c) Magnified optical image of a grid line pattern with a $50 \mu \mathrm{m}$ spacing and d-e) SEM micrographs showing the micro-structure of a patterned SWCNT-TCF. The narrow, non-perpendicular lines visible in c-d) originate from the slight shear stress induced by the filter holder sealing mechanism, leading in to hairline cracks in the grid-patterning photoresist layer and subsequently to a slight reduction of the maximum transparency of the SWCNT-TCF. 

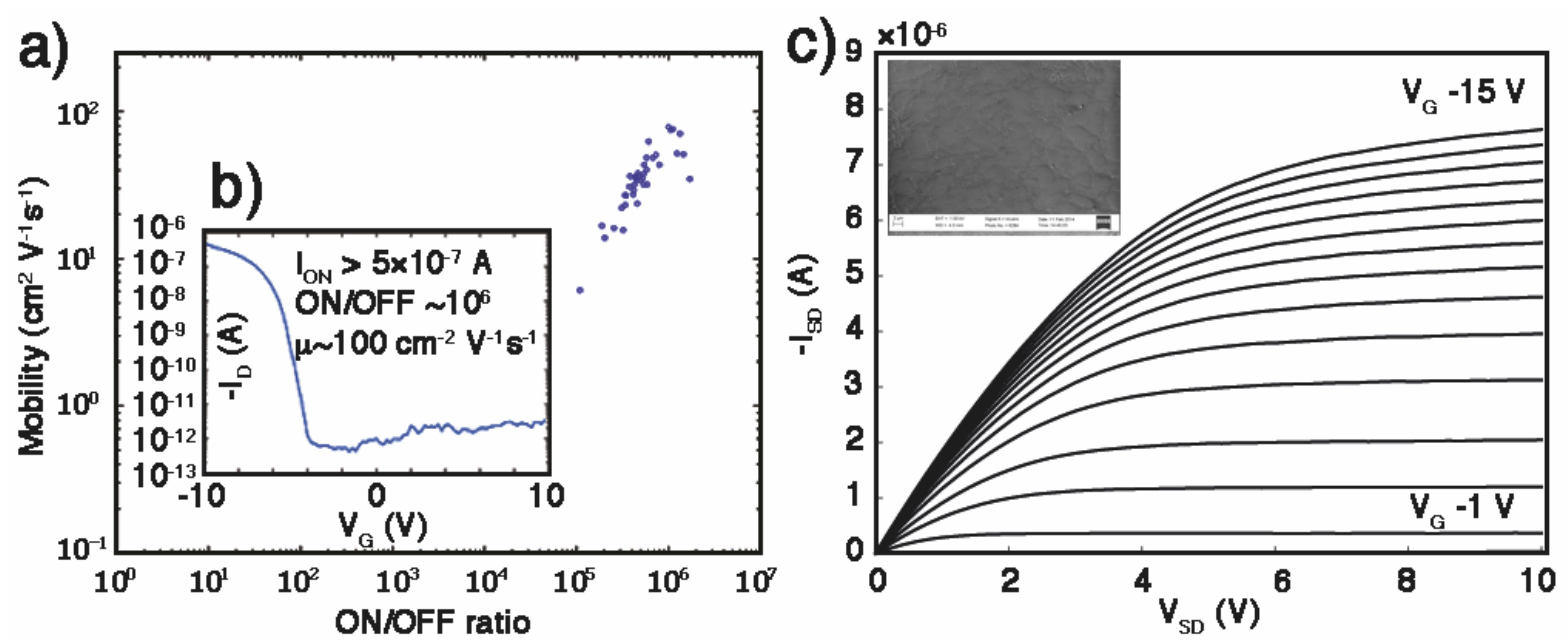

Figure 5. a) The SWCNTs synthesized at low concentration conditions exhibit excellent thin film transistors characteristics with high ON/OFF current ratios up to $10^{6}$ and mobilities up to $100 \mathrm{~cm}^{2} \mathrm{~V}^{-1} \mathrm{~s}^{-1}$, while using $\mathrm{V}_{\mathrm{DS}}$ of $-1.0 \mathrm{~V}$. Inset b) Typical transfer characteristics for SWCNT TFTs exhibit clear p-type behavior due to exposure to ambient conditions. c) Typical output ( $\mathrm{V}_{\mathrm{DS}}$ - $\mathrm{I}_{\mathrm{DS}}$ ) characteristics exhibiting linear and saturation behavior regions. Inset shows typical SWCNT network with density of $0.6 \# / \mu \mathrm{m}^{2}$ in the TFT channel area.

\section{Conclusions}

To conclude, we have demonstrated that reducing the catalyst precursor concentration introduced into a FC-CVD synthesis reactor can almost completely eliminate SWCNT bundling, simultaneously leading to the growth of longer SWCNTs. Active concentration control by real time aerosol measurements at the reactor outlet enables improved reproducibility and process stability with $<10 \%$ concentration variations over continuous 40 h synthesis runs. The increased fraction of individual SWCNTs and increase in their median length from 2.9 to $4.8 \mu$ m leads to systematically improved SWCNT transparent conductive film performance, enabling the realization of uniform SWCNT TCFs with a sheet resistance of $89 \Omega /$ sq. at $90 \%$ transmittance at the lowest concentration. The increased performance is linked to the increased length and fraction of individual SWCNTs, leading to an increased number of parallel conductive pathways when compared to heavily bundled SWCNT networks. Micro-grid patterned SWCNT TCFs further improved the performance of flexible SWCNT TCFs up to $69 \Omega /$ sq. at $97 \%$ transmittance, which is the highest reported performance for any carbon nanotube based TCF. The individual SWCNTs also made for excellent thin film transistor materials, yielding TFT-arrays with mobilities up to $100 \mathrm{~cm}^{2} \mathrm{~V}^{-}$ 
${ }^{1} \mathrm{~S}^{-1}$ and ON/OFF ratios up to $10^{6}$, demonstrating the potential of FC-CVD synthesis of individual SWCNTs for the fabrication of active transistor based electronics, and the obtained concentration control enables further studies on the impact of bundling for SWCNT TFT performance in future. To summarize, our results clearly show that control and elimination of bundling are essential for optimizing SWCNT network performance. Therefore characterization and optimization of mesoscale-parameters, particularly bundle vs. individual SWCNT fractions and structure lengths, are essential for future experimental work aiming to overcome performance limits in structures as complex as SWCNT networks.

\section{Acknowledgements}

The work has been supported by Aalto Energy Efficiency (AEF) program through the MOPPI project, by JST-EC DG RTD Coordinated Research Project IRENA, by Academy of Finland funded HISCON project and by TEKES project CARLA. T.S. was supported by the Austrian Science Fund (FWF) through grant P 28322-N36. The support by Finnish Foundation for Technology Promotion is gratefully acknowledged. This work made use of the Aalto University Nanomicroscopy Center (Aalto-NMC) premises. Aalto NanoFab (Micronova) clean room resources are greatly appreciated. Mr. Michael Treacy is acknowledged for his comments to the manuscript.

\section{References}

[1] Z. Wu, “Transparent, Conductive Carbon Nanotube Films,” Science, vol. 305, no. 5688, pp. 1273-1276, Aug. 2004.

[2] M. Kaempgen, G. S. Duesberg, and S. Roth, “Transparent carbon nanotube coatings," Applied Surface Science, vol. 252, no. 2, pp. 425-429, Oct. 2005.

[3] G. Gruner, "Carbon nanotube films for transparent and plastic electronics," J. Mater. Chem., vol. 16, no. 35, p. 3533, 2006.

[4] H.-Z. Geng, K. K. Kim, K. P. So, Y. S. Lee, Y. Chang, and Y. H. Lee, "Effect of Acid Treatment on Carbon Nanotube-Based Flexible Transparent Conducting Films,” J. Am. Chem. Soc., vol. 129, no. 25, pp. 7758-7759, Jun. 2007.

[5] B. Dan, G. C. Irvin, and M. Pasquali, "Continuous and Scalable Fabrication of Transparent Conducting Carbon Nanotube Films,” ACS Nano, vol. 3, no. 4, pp. 835843, Apr. 2009. 
[6] A. Kaskela, A. G. Nasibulin, M. Y. Timmermans, B. Aitchison, A. Papadimitratos, Y. Tian, Z. Zhu, H. Jiang, D. P. Brown, A. Zakhidov, and E. I. Kauppinen, “AerosolSynthesized SWCNT Networks with Tunable Conductivity and Transparency by a Dry Transfer Technique,” Nano Lett., vol. 10, no. 11, pp. 4349-4355, Nov. 2010.

[7] A. G. Nasibulin, A. Kaskela, K. Mustonen, A. S. Anisimov, V. Ruiz, S. Kivistö, S. Rackauskas, M. Y. Timmermans, M. Pudas, B. Aitchison, M. Kauppinen, D. P. Brown, O. G. Okhotnikov, and E. I. Kauppinen, "Multifunctional Free-Standing Single-Walled Carbon Nanotube Films,” ACS Nano, vol. 5, no. 4, pp. 3214-3221, Apr. 2011.

[8] S. J. Tans, A. R. Verschueren, and C. Dekker, "Room-temperature transistor based on a single carbon nanotube,” Nature, vol. 393, no. 6680, pp. 49-52, 1998.

[9] A. Javey, J. Guo, Q. Wang, M. Lundstrom, and H. Dai, "Ballistic carbon nanotube fieldeffect transistors,” Nature, vol. 424, no. 6949, pp. 654-657, 2003.

[10] E. Bekyarova, M. E. Itkis, N. Cabrera, B. Zhao, A. Yu, J. Gao, and R. C. Haddon, “Electronic Properties of Single-Walled Carbon Nanotube Networks,” J. Am. Chem. Soc., vol. 127, no. 16, pp. 5990-5995, Apr. 2005.

[11] C. Wang, J. Zhang, and C. Zhou, "Macroelectronic Integrated Circuits Using HighPerformance Separated Carbon Nanotube Thin-Film Transistors,” ACS Nano, vol. 4, no. 12, pp. 7123-7132, Dec. 2010.

[12] D.-M. Sun, M. Y. Timmermans, Y. Tian, A. G. Nasibulin, E. I. Kauppinen, S. Kishimoto, T. Mizutani, and Y. Ohno, "Flexible high-performance carbon nanotube integrated circuits,” Nature Nanotech, vol. 6, no. 3, pp. 156-161, 2011.

[13] D.-M. Sun, M. Y. Timmermans, A. Kaskela, A. G. Nasibulin, S. Kishimoto, T. Mizutani, E. I. Kauppinen, and Y. Ohno, "Mouldable all-carbon integrated circuits," Nature Communications, vol. 4, pp. 1-8, Aug. 2013.

[14] Y. Zhou, L. Hu, and G. Grüner, “A method of printing carbon nanotube thin films,” Appl. Phys. Lett., vol. 88, no. 12, p. 123109, 2006.

[15] P. Chen, Y. Fu, R. Aminirad, C. Wang, J. Zhang, K. Wang, K. Galatsis, and C. Zhou, "Fully Printed Separated Carbon Nanotube Thin Film Transistor Circuits and Its Application in Organic Light Emitting Diode Control,” Nano Lett., vol. 11, no. 12, pp. 5301-5308, Dec. 2011.

[16] P. H. Lau, K. Takei, C. Wang, Y. Ju, J. Kim, Z. Yu, T. Takahashi, G. Cho, and A. Javey, "Fully Printed, High Performance Carbon Nanotube Thin-Film Transistors on Flexible Substrates,” Nano Lett., vol. 13, no. 8, pp. 3864-3869, Aug. 2013. 
[17] J. Prasek, J. Drbohlavova, J. Chomoucka, J. Hubalek, O. Jasek, V. Adam, and R. Kizek, “Methods for carbon nanotubes synthesis_review,” J. Mater. Chem., vol. 21, no. 40, p. 15872, 2011.

[18] J.-H. Shin, D. W. Shin, S. P. Patole, J. H. Lee, S. M. Park, and J. B. Yoo, "Smooth, transparent, conducting and flexible SWCNT films by filtration-wet transfer processes,” J. Phys. D: Appl. Phys., vol. 42, no. 4, p. 045305, Jan. 2009.

[19] T. V. Sreekumar, T. Liu, S. Kumar, L. M. Ericson, R. H. Hauge, and R. E. Smalley, “Single-Wall Carbon Nanotube Films,” Chem. Mater., vol. 15, no. 1, pp. 175-178, Jan. 2003.

[20] P. N. Nirmalraj, P. E. Lyons, S. De, J. N. Coleman, and J. J. Boland, "Electrical Connectivity in Single-Walled Carbon Nanotube Networks,” Nano Lett., vol. 9, no. 11, pp. 3890-3895, Nov. 2009.

[21] M. S. Arnold, S. I. Stupp, and M. C. Hersam, "Enrichment of Single-Walled Carbon Nanotubes by Diameter in Density Gradients,” Nano Lett., vol. 5, no. 4, pp. 713-718, Apr. 2005.

[22] G. S. Tulevski, A. D. Franklin, and A. Afzali, "High Purity Isolation and Quantification of Semiconducting Carbon Nanotubes viaColumn Chromatography,” ACS Nano, vol. 7, no. 4, pp. 2971-2976, Apr. 2013.

[23] A. Moisala, A. G. Nasibulin, D. P. Brown, H. Jiang, L. Khriachtchev, and E. I. Kauppinen, "Single-walled carbon nanotube synthesis using ferrocene and iron pentacarbonyl in a laminar flow reactor," Chemical Engineering Science, vol. 61, no. 13, pp. 4393-4402, Jul. 2006.

[24] A. Znidarsic, A. Kaskela, P. laiho, M. Gaberscek, Y. Ohno, A. G. Nasibulin, E. I. Kauppinen, and A. Hassanien, "Spatially Resolved Transport Properties of Pristine and Doped Single-Walled Carbon Nanotube Networks,” J. Phys. Chem. C, vol. 117, no. 25, pp. 13324-13330, Jun. 2013.

[25] S. Seppälä, E. Häkkinen, M. J. Alava, V. Ermolov, and E. T. Seppälä, "Electrical transport properties of percolating random networks of carbon nanotube bundles,” EPL, vol. 91, no. 4, p. 47002, Sep. 2010.

[26] W. C. Hinds, “Aerosol technology: properties, behavior, and measurement of airborne particles," 2012.

[27] D.-W. Shin, J. H. Lee, Y.-H. Kim, S. M. Yu, S.-Y. Park, and J.-B. Yoo, “A role of HNO3 on transparent conducting film with single-walled carbon nanotubes," Nanotechnology, vol. 20, no. 47, p. 475703, Oct. 2009. 
[28] N. Fukaya, D. Y. Kim, S. Kishimoto, S. Noda, and Y. Ohno, “One-Step Sub-10 $\mu \mathrm{m}$ Patterning of Carbon-Nanotube Thin Films for Transparent Conductor Applications,” ACS Nano, p. 140401093137001 , Apr. 2014.

[29] S. J. Kang, C. Kocabas, T. Ozel, M. Shim, N. Pimparkar, M. A. Alam, S. V. Rotkin, and J. A. Rogers, "High-performance electronics using dense, perfectly aligned arrays of single-walled carbon nanotubes,” Nature Nanotech, vol. 2, no. 4, pp. 230-236, Mar. 2007.

[30] H. W. C. Postma, A. Sellmeijer and C. Deeker, "Manipulation and Imaging of Individual Single-Walled Carbon Nanotubes with an Atomic Force Microscope," Advanced Materials, vol. 12, no. 17, September 2000. 